\title{
Self-torque and angular momentum balance for a spinning charged sphere
}

\author{
Béatrice Bonga \\ Perimeter Institute for Theoretical Physics, Waterloo, Ontario N2L 2Y5, Canada \\ Eric Poisson \\ Department of Physics, University of Guelph, Guelph, Ontario, N1G 2W1, Canada \\ Huan Yang \\ Department of Physics, University of Guelph, Guelph, Ontario, N1G 2W1, Canada and \\ Perimeter Institute for Theoretical Physics, Waterloo, Ontario N2L 2Y5, Canada
}

(Dated: August 27, 2018)

\begin{abstract}
Angular momentum balance is examined in the context of the electrodynamics of a spinning charged sphere, which is allowed to possess any variable angular velocity. We calculate the electric and magnetic fields of the (hollow) sphere, and express them as expansions in powers of $\tau / t_{c} \ll 1$, the ratio of the light-travel time $\tau$ across the sphere and the characteristic time scale $t_{c}$ of variation of the angular velocity. From the fields we compute the self-torque exerted by the fields on the sphere, and argue that only a piece of this self-torque can be associated with radiation reaction. Then we obtain the rate at which angular momentum is radiated away by the shell, and the total angular momentum contained in the electromagnetic field. With these results we demonstrate explicitly that the field angular momentum is lost in part to radiation and in part to the self-torque; angular momentum balance is thereby established. Finally, we examine the angular motion of the sphere under the combined action of the self-torque and an additional torque supplied by an external agent.
\end{abstract}

\section{INTRODUCTION AND SUMMARY}

The aim of this paper is to investigate the electrodynamics of a spinning charged sphere. Our wish is to bring together two themes of the standard textbook literature, one rather neglected, the other shrouded in mystery.

The neglected theme is electromagnetic angular momentum, which tends to be mentioned in passing in most texts, but rarely explored beyond a very small number of examples. For example, Griffiths [1] introduces the field angular momentum in Sec. 8.2.4 and computes it explicitly for a solenoid encased between two cylindrical shells 1 Apart from another appearance in Problem 8.17, this is the sole mention of electromagnetic angular momentum in this popular text. As another example, Jackson [6] mentions the field angular momentum on a few occasions, but the topic is not deemed worthy of a section of its own.

The theme shrouded in mystery is self-force and radiation reaction. Despite valiant attempts to elucidate this topic in the standard texts (see, for example, Sec. 11.2.2 in Griffiths, or Chapter 16 of Jackson), these aspects of electromagnetic theory remain conceptually challenging to most students (and instructors), thanks to the lurking infinities, mass renormalization, runaway solutions, pre-acceleration, and the like. This topic has been the subject of a vast literature, and American Journal of Physics has been an active participant. A simple pedagogical introduction to the subject can be found in Boyer's paper [7], and a useful historical survey was provided by Rohrlich [8]. In Ref. [9] Griffiths, Proctor, and Schroeter compare the predictions of the standard equation of motion for a charged particle, the Abraham-Lorentz equation, to those of the less pathological Landau-Lifshitz equation (see Sec. 75 of Ref. [10]). The issue of mass renormalization is discussed by Griffiths and Owen [11]. A clever derivation of the self-force was devised by Griffiths and Szeto [12] on the basis of a dumbbell model for an electric charge; their construction was revisited and generalized by Ori and Rosenthal [13, 14].

In our opinion, the conceptual difficulties associated with self-force and radiation reaction stem from a misguided insistence that the Maxwell-Lorentz theory should apply to a point particle. While the electromagnetic field of a point particle is perfectly well defined, its action back on the particle is not, and much contortion is required to obtain self-consistent equations of motion for the particle. Our point of view is that in the classical framework of the Maxwell-Lorentz theory, equations of motion should only be formulated for extended blobs of charge, and a pointlike limit should be recovered only when the electromagnetic field varies on a length scale that is long compared with the size of the blob. In this context the equations of motion are necessarily approximate, and self-consistency can be achieved only within the limits of the approximation. For a rigorous implementation of this idea, see Ref. [15].

\footnotetext{
1 This variant of Feynman's disk paradox was first proposed by Romer [2] and Boos [3]; the original paradox is discussed in Sec. 17.4 of Volume II of the Feynman lectures [4], and in Ref. [5].
} 
Our interest in this paper is with the electrodynamics of an infinitely thin, spherical shell that is uniformly charged and spinning on a central axis with a variable angular velocity. The spinning motion of the sphere implies that angular momentum shall be one of its most important attributes, and thus is the first theme introduced 2 The fact that the angular velocity is variable implies that the shell emits electromagnetic radiation, which takes angular momentum away from the system; this loss must be balanced by a self-torque acting on the shell, and we have our second theme. Such issues were explored previously in a paper by Stump and Pollack [17], who calculated the self-torque acting on a point magnetic dipole. We feel that the spinning shell, with its finite extension, provides a much more satisfactory starting point for this discussion.

The system's angular-momentum vector $\boldsymbol{J}$ is subjected to two conservation equations, which are established in Sec. III The first concerns $\boldsymbol{J}^{\mathrm{em}}$, the electromagnetic piece of the angular momentum, and it reads

$$
\frac{d}{d t} J^{\mathrm{em}}=-\mathcal{N}^{\mathrm{em}}-\mathfrak{T}^{\mathrm{em}}
$$

where $\mathcal{N}^{\mathrm{em}}$ is the rate at which angular momentum is radiated away, and $\mathcal{T}^{\mathrm{em}}$ is the electromagnetic torque - the self-torque - acting on the shell. The equation states that the field angular momentum is communicated partly to the radiation and partly to the shell. The second conservation statement concerns $\boldsymbol{J}^{\text {mech }}$, the shell's mechanical angular momentum, and it reads

$$
\frac{d}{d t} J^{\text {mech }}=\mathcal{T}^{\mathrm{em}}+\mathcal{T}^{\mathrm{ext}}
$$

where $\mathcal{T}^{\mathrm{em}}$ is again the self-torque, and $\mathfrak{T}^{\text {ext }}$ is the torque supplied by an external agent acting on the shell. The equation states that the shell's angular momentum changes because of the combined action of both torques. The sum of $\boldsymbol{J}^{\mathrm{em}}$ and $\boldsymbol{J}^{\mathrm{mech}}$ is the total angular momentum $\boldsymbol{J}$, and according to Eqs. (1.1) and (1.2), it satisfies the conservation statement $d \boldsymbol{J} / d t=-\mathcal{N}^{\mathrm{em}}-\mathcal{T}^{\text {ext }}$. The total angular momentum is not conserved, because of radiative losses and the torque exerted by the external agent.

The conservation statement of Eq. (1.1) can be verified by calculating the field angular momentum, the radiated losses, and the self-torque. This requires expressions for the shell's electric and magnetic fields at any time $t$ and any position $\boldsymbol{x}$. Provided that we introduce a mild and sensible assumption, the fields of a spinning charged sphere can be calculated for any variable angular velocity. The assumption is that the angular velocity is taken to vary on a characteristic time scale $t_{c}$ that is very long compared with $\tau=R / c$, the light-travel time across (half) the sphere; $R$ is the sphere's radius and $c$ is the speed of light. The fields are calculated in Sec. III and presented as expansions in powers of $\tau / t_{c} \ll 1$. In principle, these can be carried out to an arbitrarily high order; we truncate them after a representative number of terms.

With the fields in hand, we calculate the self-torque in Sec. IV and express it also as an expansion in powers of $\tau / t_{c}$. We find that only pieces of the self-torque can be associated with radiation reaction; the remaining pieces (which include the dominant contribution to the self-torque) have nothing to do with radiation reaction. As we explain in Sec. IV] the self-torque can be decomposed into terms that come with even powers of $\tau / t_{c}$ (and therefore even powers of $c$ ), and terms that come with odd powers of $\tau / t_{c}$ (and therefore odd powers of $c$ ). The odd terms are sensitive to the choice of boundary condition at infinity; they change sign when the retarded solution describing an outgoing wave is switched to the advanced solution describing an incoming wave. These odd terms, therefore, can be associated with radiation reaction, with the justification that a switch of boundary condition will produce a change in the self-torque. The even terms, on the other hand, are insensitive to the choice of boundary condition, and stay the same after a switch; these terms have nothing to do with radiation reaction. The dominant contribution to the self-torque is even in $\tau / t_{c}$.

The radiated angular momentum is calculated in Sec. $\mathrm{V}$, and presented as an expansion in powers of $\tau / t_{c}$. As can be expected from the preceding discussion, each term is odd in $c$, and therefore sensitive to the choice of boundary condition at infinity. We note that the radiated angular momentum (like the self-torque) is proportional to $q$, the total charge of the spinning sphere. This provides a vivid illustration of the fact that the flux of angular momentum depends not only on the radiative, $1 / r$ part of the electromagnetic field, but also on its Coulombic, $1 / r^{2}$ part; this point was made forcibly in a recent paper by Ashtekar and Bonga [18]. The field's angular momentum is calculated in Sec. VI and Eq. (1.1) is verified explicitly.

In Sec. VII we turn to the shell's angular motion, governed by Eq. (1.2). We imagine that an external agent applies a torque on the shell, in addition to the self-torque supplied by the electromagnetic field. The resulting equation of motion is again presented as an expansion in powers of $\tau / t_{c}$, with the external torque giving the dominant contribution,

\footnotetext{
2 The electromagnetic angular momentum of a spinning shell with constant angular velocity was previously calculated by de Castro 16$]$.
} 
and the self-torque splitting up into radiation-reaction terms (odd in $\tau / t_{c}$ ) and terms (even in $\tau / t_{c}$ ) that have nothing to do with radiation reaction. A feature of the equation of motion is that it is written in terms of an effective mass $M_{\text {eff }}$, the sum of the shell's mass and the additional inertia contributed by the electrostatic field; this is a form of mass renormalization, a ubiquitous procedure of self-force calculations. Another feature of the equation of motion is that it requires a reduction of order (see Sec. 75 of Ref. [10] or Ref. [19]) to avoid the emergence of runaway solutions. In our case this procedure is natural and justified on the grounds of the underlying assumption that $\tau / t_{c} \ll 1$. We examine several examples of the shell's angular motion, corresponding to different external torques.

The electrodynamics of a spinning charged sphere unlocks a number of important lessons. First, it presents an excellent opportunity to reflect on the role of angular momentum in electromagnetism, a largely neglected subject. Second, it provides a vivid example of the fact that the radiated angular momentum may not solely depend on the $1 / r$, radiative part of the electromagnetic field, but can also depend on the $1 / r^{2}$, Coulombic part. Third, it provides a conceptually clean setup to understand how the field can act back on its source; the self-torque is well defined and can be computed explicitly, and this should help lift some of the aura of mystery that tends to accompany this topic. Fourth, it reveals that the notions of self-torque and radiation reaction must be clearly distinguished. Indeed, the self-torque contains two types of terms: time-antisymmetric terms that reflect the choice of outgoing-wave boundary condition and can therefore be associated with radiation reaction, and time-symmetric terms that are insensitive to the choice of boundary condition and have therefore nothing to do with radiation reaction. And fifth, it provides an instance in which the equation of motion for the source of an electromagnetic field is well defined and can be solved explicitly. These lessons can all be learned at a reasonable cost: All calculations are straightforward and accessible to a starting graduate student, and they might also be tractable to a well-educated senior undergraduate (instructors should be able to cope as well). We believe that these lessons are well worth learning, and that this paper could form the basis of an independent study module in a graduate course in electromagnetism.

This paper is dedicated to the memory of Steve Detweiler, who understood better than most the power of simple models to foster understanding of complicated things.

\section{CONSERVATION STATEMENTS}

In this section we establish a number of conservation statements relating to the momentum and angular momentum of an electromagnetic field. These are formulated in some generality, with the assumption that the field is sourced by a bounded charge distribution supported by a fluid; for simplicity we take the fluid to be electrically and magnetically unpolarized. The conservation laws will then apply, as a special case, to the field sourced by a spinning charged sphere.

Throughout the paper we denote three-dimensional vectors with a bold-italic symbol such as $\boldsymbol{E}$, and denote individual components with $E_{a}$ or $(\boldsymbol{E})_{a}$. We shall move freely between the notations, making the most convenient choice given the context. We adopt the Einstein summation convention, according to which repeated vector (or tensor) indices are summed over. As examples of our notation, we write $|\boldsymbol{E}|^{2}=\boldsymbol{E} \cdot \boldsymbol{E}=E_{a} E_{a}$ and $(\boldsymbol{E} \times \boldsymbol{B})_{a}=\epsilon_{a b c} E_{b} B_{c}$, where $\epsilon_{a b c}$ is the completely antisymmetric permutation symbol. We let $\partial_{t}:=\partial / \partial t$ stand for partial differentiation with respect to time $t$, and $\partial_{a}:=\partial / \partial x^{a}$ for partial differentiation with respect to the spatial coordinate $x^{a}$.

We begin with a conservation statement for the linear momentum contained in the electromagnetic field. Let

$$
g_{a}^{\mathrm{em}}:=\epsilon_{0}(\boldsymbol{E} \times \boldsymbol{B})_{a}
$$

be the field's momentum density, and

$$
T_{a b}^{\mathrm{em}}:=-\epsilon_{0}\left(E_{a} E_{b}-\frac{1}{2} \delta_{a b}|\boldsymbol{E}|^{2}\right)-\frac{1}{\mu_{0}}\left(B_{a} B_{b}-\frac{1}{2} \delta_{a b}|\boldsymbol{B}|^{2}\right)
$$

be the field's stress tensor, defined such that $T_{a b}^{\mathrm{em}} d S_{b}$ is the momentum crossing out of an element of surface $d S_{b}$ per unit time 3 Finally, let

$$
f_{a}^{\mathrm{em}}:=\rho E_{a}+(\boldsymbol{j} \times \boldsymbol{B})_{a}
$$

be the Lorentz force density on a charge distribution described by the charge density $\rho$ and current density $\boldsymbol{j}$. Then the conservation statement

$$
\partial_{t} g_{a}^{\mathrm{em}}+\partial_{b} T_{a b}^{\mathrm{em}}=-f_{a}^{\mathrm{em}}
$$

\footnotetext{
3 The stress tensor is sometimes defined with plus signs, with the alternative convention that $T_{a b}^{\mathrm{em}} d S_{b}$ is the momentum crossing into the element of surface per unit time.
} 
follows as a consequence of Maxwell's equations. This is the momentum theorem for the electromagnetic field, which is proved in the standard texts (see, for example, Sec. 8.2.2 of Griffiths [1] or Sec. 6.7 of Jackson [6]).

The momentum theorem gives rise to an angular-momentum theorem, which we formulate in integral form. Let $V$ be a region of three-dimensional space bounded by a closed two-surface $S$. We assume that $V$ and $S$ are fixed in space. Let

$$
J_{a}^{\mathrm{em}}:=\int_{V}\left(\boldsymbol{x} \times \boldsymbol{g}^{\mathrm{em}}\right)_{a} d^{3} x
$$

be the total angular momentum of the electromagnetic field in $V ; \boldsymbol{x}$ denotes the position vector. Then a consequence of Eq. (2.4) is the conservation statement

$$
\frac{d}{d t} J_{a}^{\mathrm{em}}=-\mathcal{N}_{a}^{\mathrm{em}}-\mathcal{T}_{a}^{\mathrm{em}}
$$

where

$$
\mathcal{N}_{a}^{\mathrm{em}}:=\epsilon_{a b c} \oint_{S} x_{b} T_{c d}^{\mathrm{em}} d S_{d}
$$

is the field angular momentum crossing out of $S$ per unit time, and

$$
\mathcal{T}_{a}^{\mathrm{em}}:=\epsilon_{a b c} \int_{V} x_{b} f_{c}^{\mathrm{em}} d^{3} x
$$

is the torque exerted by the fields on the charge distribution in $V$. Equation (2.6) states that the rate at which the field angular momentum leaves $V$ is equal to the rate at which it crosses out of $S$ added to the torque exerted on all charges within $V$. The equation is established by taking the time derivative inside the integral in Eq. (2.5), substituting Eq. (2.4), and integrating by parts.

The fluid that supports the charge distribution contributes mechanical momentum and angular momentum. Let $\rho_{\mathrm{m}}$ be the fluid's mass density, and let $v_{a}$ be its velocity field. Mass conservation is expressed by the continuity equation $\partial_{t} \rho_{\mathrm{m}}+\partial_{a}\left(\rho_{\mathrm{m}} v_{a}\right)=0$, and in the absence of charges and fields, the fluid is subjected to the momentum theorem

$$
\partial_{t} g_{a}^{\text {mech }}+\partial_{b} T_{a b}^{\text {mech }}=f_{a}^{\mathrm{ext}}
$$

where $g_{a}^{\text {mech }}=\rho_{\mathrm{m}} v_{a}$ is the mechanical momentum density, $T_{a b}^{\text {mech }}$ the mechanical stress tensor, and $f_{a}^{\text {ext }}$ the force density supplied by external agents. In the case of a perfect fluid, for example, the mechanical stress tensor is given by $T_{a b}^{\text {mech }}=\rho_{\mathrm{m}} v_{a} v_{b}+p \delta_{a b}$, where $p$ is the pressure, and the momentum theorem becomes $\rho_{\mathrm{m}} d v_{a} / d t+\partial_{a} p=f_{a}^{\text {ext }}$. This is recognized as Euler's equation.

If we now allow the fluid to be charged, and allow for the presence of an electromagnetic field, the momentum theorem becomes

$$
\partial_{t} g_{a}+\partial_{b} T_{a b}=f_{a}^{\mathrm{ext}}
$$

where $g_{a}:=g_{a}^{\mathrm{mech}}+g_{a}^{\mathrm{em}}$ is the total momentum density, $T_{a b}:=T_{a b}^{\mathrm{mech}}+T_{a b}^{\mathrm{em}}$ the total stress tensor, and $f_{a}^{\mathrm{ext}}$ the force density provided by external agents (excluding the Lorentz force density). In the case of a perfect fluid, the momentum theorem becomes $\rho_{\mathrm{m}} d v_{a} / d t+\partial_{a} p-f_{a}^{\mathrm{em}}=f_{a}^{\text {ext }}$, Euler's equation for a charged fluid.

The momentum theorem for a charged fluid gives rise to a conservation statement for the total angular momentum contained in the region $V$,

$$
J_{a}:=\int_{V}(\boldsymbol{x} \times \boldsymbol{g})_{a} d^{3} x=J_{a}^{\mathrm{mech}}+J_{a}^{\mathrm{em}} .
$$

We assume that the fluid occupies a bounded region within $V$, and that this region does not extend all the way to $S$. In this case $T_{a b}$ evaluated on $S$ consists of a field contribution only, and the mechanical contribution vanishes. The conservation statement is

$$
\frac{d}{d t} J_{a}=-\mathcal{N}_{a}^{\mathrm{em}}-\mathcal{T}_{a}^{\mathrm{ext}}
$$

where $\mathcal{N}_{a}^{\mathrm{em}}$ is the field angular momentum crossing out of $S$ per unit time, as given by Eq. (2.7), and

$$
\mathcal{T}_{a}^{\text {ext }}:=\epsilon_{a b c} \int_{V} x_{b} f_{c}^{\text {ext }} d^{3} x
$$


is the torque supplied by the external agents.

Equations (2.6) and (2.12) imply

$$
\frac{d}{d t} J_{a}^{\mathrm{mech}}=\mathcal{T}_{a}^{\mathrm{em}}+\mathcal{T}_{a}^{\mathrm{ext}}
$$

the statement that the mechanical angular momentum changes because of the combined action of the electromagnetic and external torques.

\section{FIELDS OF A SPINNING CHARGED SPHERE}

We consider an infinitely thin, spherical shell of radius $R$ spinning on a central axis with a time-changing angular velocity $\Omega(t)$. The shell possesses a tangential pressure that supports it against gravity, and we assume that its material is not polarized (either electrically or magnetically) by the electromagnetic field. We align the $z$-direction with the rotation axis, and employ the usual spherical coordinates $(r, \theta, \phi)$ in addition to the Cartesian coordinates $(x, y, z)$. Each coordinate system comes with a basis of unit vectors. For the Cartesian coordinates we have $(\hat{\boldsymbol{x}}, \hat{\boldsymbol{y}}, \hat{\boldsymbol{z}})$, and for the spherical coordinates we have $(\hat{\boldsymbol{r}}, \hat{\boldsymbol{\theta}}, \hat{\boldsymbol{\phi}})$.

The shell carries a charge $q$, and its charge density is given by

$$
\rho=\frac{q}{4 \pi R^{2}} \delta(r-R) .
$$

The velocity of an element of charge on the shell is $\boldsymbol{v}=R \Omega(t) \sin \theta \hat{\boldsymbol{\phi}}$. The current density is $\boldsymbol{j}=\rho \boldsymbol{v}$, and the shell's magnetic moment is

$$
\boldsymbol{m}:=\frac{1}{2} \int \boldsymbol{x} \times \boldsymbol{j} d^{3} x=\frac{1}{3} q R^{2} \Omega(t) \hat{\boldsymbol{z}} .
$$

In the following we shall express $\Omega(t)$ in terms of $m(t)$, with $m:=|\boldsymbol{m}|=\frac{1}{3} q R^{2} \Omega$.

In the usual Lorenz gauge, the potentials created by the spinning shell satisfy wave equations, with solutions

$$
\begin{aligned}
\Phi(t, \boldsymbol{x}) & =\frac{1}{4 \pi \epsilon_{0}} \int \rho\left(t^{\prime}, \boldsymbol{x}^{\prime}\right) G\left(t, \boldsymbol{x} ; t^{\prime}, \boldsymbol{x}^{\prime}\right) d t^{\prime} d^{3} x^{\prime}, \\
A_{a}(t, \boldsymbol{x}) & =\frac{\mu_{0}}{4 \pi} \int j_{a}\left(t^{\prime}, \boldsymbol{x}^{\prime}\right) G\left(t, \boldsymbol{x} ; t^{\prime}, \boldsymbol{x}^{\prime}\right) d t^{\prime} d^{3} x^{\prime},
\end{aligned}
$$

where $G\left(t, \boldsymbol{x} ; t^{\prime}, \boldsymbol{x}^{\prime}\right)$ is the retarded Green's function for the wave equation. Because $\rho$ is static and spherically symmetric, the scalar potential is given simply by

$$
\begin{gathered}
\Phi_{\text {in }}=\frac{1}{4 \pi \epsilon_{0}} \frac{q}{R}, \\
\Phi_{\text {out }}=\frac{1}{4 \pi \epsilon_{0}} \frac{q}{r},
\end{gathered}
$$

with $\Phi_{\text {in }}$ applying when $r<R$, and $\Phi_{\text {out }}$ when $r>R$.

Because $j_{a}$ is time dependent (via $m$ ), the computation of the vector potential is more involved. It is helpful to work with $A_{y}-i A_{x}$, noting that $j_{z}=0$ and $j_{y}-i j_{x} \propto \sin \theta e^{i \phi}$, a spherical harmonic with labels $\ell=1$ and $\mathrm{m}=1$. We take advantage of a decomposition of the Green's function in spherical harmonics, provided by

$$
G\left(t, \boldsymbol{x} ; t^{\prime}, \boldsymbol{x}^{\prime}\right)=\sum_{\ell \mathrm{m}} g_{\ell}\left(t, r ; t^{\prime}, r^{\prime}\right) Y_{\ell \mathrm{m}}^{*}\left(\theta^{\prime}, \phi^{\prime}\right) Y_{\ell \mathrm{m}}(\theta, \phi),
$$

where $(r, \theta, \phi)$ are the spherical coordinates attached to $\boldsymbol{x}$, while $\left(r^{\prime}, \theta^{\prime}, \phi^{\prime}\right)$ are those attached to $\boldsymbol{x}^{\prime}$, and where

$$
g_{\ell}\left(t, r ; t^{\prime}, r^{\prime}\right)=\frac{2 \pi c}{r r^{\prime}} \Theta\left(\Delta-\left|r-r^{\prime}\right|\right) \Theta\left(r+r^{\prime}-\Delta\right) P_{\ell}(\xi),
$$

with $\Theta(u)$ denoting the step function, $P_{\ell}(\xi)$ the Legendre polynomials, $\Delta:=c\left(t-t^{\prime}\right)$, and

$$
\xi:=\frac{r^{2}+r^{\prime 2}-\Delta^{2}}{2 r r^{\prime}}
$$


Because this decomposition does not seem to be widely known, but can be immensely useful, we provide a derivation in the Appendix.

We insert Eq. (3.5) within the integral for $A_{y}-i A_{x}$, write $d^{3} x^{\prime}=r^{\prime 2} d r^{\prime} d \Omega^{\prime}$ where $d \Omega^{\prime}:=\sin \theta^{\prime} d \theta^{\prime} d \phi^{\prime}$, perform the angular integration by exploiting the orthonormality of spherical harmonics, and evaluate the radial integral with the help of the delta function contained in $\boldsymbol{j}$. We arrive at

$$
\boldsymbol{A}=\frac{\mu_{0}}{4 \pi} \Gamma(t, r) \sin \theta \hat{\boldsymbol{\phi}}
$$

where

$$
\Gamma(t, r):=\frac{3}{4 \pi R} \int m\left(t^{\prime}\right) g_{1}\left(t, r ; t^{\prime}, R\right) d t^{\prime} .
$$

Inserting Eq. (3.6) with $\ell=1$ and accounting for the step functions, this function becomes

$$
\Gamma(t, r)=\frac{3 c}{4 R^{3} r^{2}} \int_{t-(r+R) / c}^{t-|r-R| / c} m\left(t^{\prime}\right)\left[r^{2}+R^{2}-c^{2}\left(t-t^{\prime}\right)^{2}\right] d t^{\prime} .
$$

In principle, the integral can be evaluated only once $m\left(t^{\prime}\right)$ is specified. As we shall see, however, we can avoid making an explicit identification, and obtain $\Gamma(t, r)$ for any $m\left(t^{\prime}\right)$, at the small price of a sensible assumption. We shall assume that $t_{c}$, the characteristic time scale of variation of $m\left(t^{\prime}\right)$, is very long compared with $\tau:=R / c$, the light-travel time across (half) the sphere. The assumption implies, for example, that $\tau \dot{m} \ll m$, that $\tau^{2} \ddot{m} \ll \tau \dot{m}$, and so on, with overdots indicating differentiation with respect to time.

When $r<R$, the bounds of integration in Eq. (3.10) become $t-R / c-r / c$ and $t-R / c+r / c$, respectively, and the integration takes place over a short time interval $2 r / c<2 \tau$ around $t=R / c$. Over this interval $m\left(t^{\prime}\right)$ can be expressed as a Taylor expansion about $t^{\prime}=t-R / c$, and the integral can be evaluated. We obtain

$$
\Gamma_{\text {in }}(t, r)=\frac{r}{R^{3}}\left[(m+\tau \dot{m})+\frac{1}{10} \frac{r^{2}}{c^{2}}\left(m^{(2)}+\tau m^{(3)}\right)+\frac{1}{280} \frac{r^{4}}{c^{4}}\left(m^{(4)}+\tau m^{(5)}\right)+\frac{1}{15120} \frac{r^{6}}{c^{6}}\left(m^{(6)}+\tau m^{(7)}\right)+\cdots\right]_{t-R / c},
$$

where a number within brackets in a superscript gives the number of differentiations, and the subscript after the end square bracket indicates that $m$ and its derivatives are evaluated at the time $t-R / c$. We remark that $m$ and its derivatives could be further Taylor expanded about the time $t$, but this form for $\Gamma_{\text {in }}$ happens to be convenient to work with.

When $r>R$, the bounds of integration in Eq. (3.10) become $t-r / c-R / c$ and $t-r / c+R / c$, respectively, and the integration again takes place over a short time interval $2 R / c=2 \tau$. Over this interval $m\left(t^{\prime}\right)$ can again be expressed as a Taylor expansion, this time about $t^{\prime}=t-r / c$, and the integral can again be evaluated. We obtain

$$
\Gamma_{\text {out }}(t, r)=\frac{1}{r^{2}}\left[\left(m+\frac{r}{c} \dot{m}\right)+\frac{1}{10} \tau^{2}\left(m^{(2)}+\frac{r}{c} m^{(3)}\right)+\frac{1}{280} \tau^{4}\left(m^{(4)}+\frac{r}{c} m^{(5)}\right)+\frac{1}{15120} \tau^{6}\left(m^{(6)}+\frac{r}{c} m^{(7)}\right)+\cdots\right]_{t-r / c},
$$

where $m$ and its derivatives are now evaluated at the time $t-r / c$. It is easy to verify that $\Gamma$ is continuous at $r=R$. Inserting Eqs. (3.11) and (3.12) within Eq. (3.8) gives us the vector potential of a rotating charged sphere.

With the potentials in hand it is a straightforward exercise to obtain the fields. The electric field can be decomposed into a Coulomb piece defined by $\boldsymbol{E}^{\mathrm{C}}=-\boldsymbol{\nabla} \Phi$ and an induction piece defined by $\boldsymbol{E}^{\mathrm{I}}=-\partial_{t} \boldsymbol{A}$. These are given by

$$
\begin{aligned}
\boldsymbol{E}_{\text {in }}^{\mathrm{C}}= & 0, \\
\boldsymbol{E}_{\text {in }}^{\mathrm{I}}= & -\frac{\mu_{0}}{4 \pi} \frac{r}{R^{3}}\left[(\dot{m}+\tau \ddot{m})+\frac{1}{10} \frac{r^{2}}{c^{2}}\left(m^{(3)}+\tau m^{(4)}\right)+\frac{1}{280} \frac{r^{4}}{c^{4}}\left(m^{(5)}+\tau m^{(6)}\right)\right. \\
& \left.+\frac{1}{15120} \frac{r^{6}}{c^{6}}\left(m^{(7)}+\tau m^{(8)}\right)+\cdots\right]_{t-R / c} \sin \theta \hat{\boldsymbol{\phi}}
\end{aligned}
$$

inside the sphere; we recall that $\tau=R / c$. Outside the sphere we have

$$
\begin{aligned}
& \boldsymbol{E}_{\mathrm{out}}^{\mathrm{C}}=\frac{1}{4 \pi \epsilon_{0}} \frac{q}{r^{2}} \hat{\boldsymbol{r}}, \\
& \boldsymbol{E}_{\mathrm{out}}^{\mathrm{I}}=-\frac{\mu_{0}}{4 \pi} \frac{1}{r^{2}}\left[\left(\dot{m}+\frac{r}{c} \ddot{m}\right)+\frac{1}{10} \tau^{2}\left(m^{(3)}+\frac{r}{c} m^{(4)}\right)+\frac{1}{280} \tau^{4}\left(m^{(5)}+\frac{r}{c} m^{(6)}\right)\right.
\end{aligned}
$$




$$
\left.+\frac{1}{15120} \tau^{6}\left(m^{(7)}+\frac{r}{c} m^{(8)}\right)+\cdots\right]_{t-r / c} \sin \theta \hat{\boldsymbol{\phi}}
$$

The magnetic field $\boldsymbol{B}=\boldsymbol{\nabla} \times \boldsymbol{A}=B_{r} \hat{\boldsymbol{r}}+B_{\theta} \hat{\boldsymbol{\theta}}$ has the nonvanishing components

$$
\begin{aligned}
B_{r}^{\text {in }}= & \frac{\mu_{0}}{4 \pi} \frac{2}{R^{3}}\left[(m+\tau \dot{m})+\frac{1}{10} \frac{r^{2}}{c^{2}}\left(m^{(2)}+\tau m^{(3)}\right)+\frac{1}{280} \frac{r^{4}}{c^{4}}\left(m^{(4)}+\tau m^{(5)}\right)\right. \\
& \left.+\frac{1}{15120} \frac{r^{6}}{c^{6}}\left(m^{(6)}+\tau m^{(7)}\right)+\cdots\right]_{t-R / c} \cos \theta, \\
B_{\theta}^{\text {in }}= & -\frac{\mu_{0}}{4 \pi} \frac{2}{R^{3}}\left[(m+\tau \dot{m})+\frac{1}{5} \frac{r^{2}}{c^{2}}\left(m^{(2)}+\tau m^{(3)}\right)+\frac{3}{280} \frac{r^{4}}{c^{4}}\left(m^{(4)}+\tau m^{(5)}\right)\right. \\
& \left.+\frac{1}{3780} \frac{r^{6}}{c^{6}}\left(m^{(6)}+\tau m^{(7)}\right)+\cdots\right]_{t-R / c} \sin \theta
\end{aligned}
$$

inside the sphere, and

$$
\begin{aligned}
B_{r}^{\text {out }}= & \frac{\mu_{0}}{4 \pi} \frac{2}{r^{3}}\left[\left(m+\frac{r}{c} \dot{m}\right)+\frac{1}{10} \tau^{2}\left(m^{(2)}+\frac{r}{c} m^{(3)}\right)+\frac{1}{280} \tau^{4}\left(m^{(4)}+\frac{r}{c} m^{(5)}\right)\right. \\
& \left.+\frac{1}{15120} \tau^{6}\left(m^{(6)}+\frac{r}{c} m^{(7)}\right)+\cdots\right]_{t-r / c} \cos \theta, \\
B_{\theta}^{\text {out }}= & \frac{\mu_{0}}{4 \pi} \frac{1}{r^{3}}\left[\left(m+\frac{r}{c} \dot{m}+\frac{r^{2}}{c^{2}} \ddot{m}\right)+\frac{1}{10} \tau^{2}\left(m^{(2)}+\frac{r}{c} m^{(3)}+\frac{r^{2}}{c^{2}} m^{(4)}\right)+\frac{1}{280} \tau^{4}\left(m^{(4)}+\frac{r}{c} m^{(5)}+\frac{r^{2}}{c^{2}} m^{(6)}\right)\right. \\
& \left.+\frac{1}{15120} \tau^{6}\left(m^{(6)}+\frac{r}{c} m^{(7)}+\frac{r^{2}}{c^{2}} m^{(8)}\right)+\cdots\right]_{t-r / c} \sin \theta
\end{aligned}
$$

outside the sphere.

\section{SELF-TORQUE}

The torque exerted by the electromagnetic field on the spinning sphere - the self-torque — is given by Eq. (2.8), in which we insert the Lorentz force density of Eq. (2.3). The calculation requires some care because the fields are discontinuous at $r=R$. It is easy to see, however, that the Coulomb piece of the electric field (which is discontinuous) does not contribute to the torque. The induction piece does contribute, and this contribution is unambiguous because $\boldsymbol{E}^{\mathrm{I}}$ happens to be continuous at $r=R$. The magnetic field is discontinuous, but calculation shows that the magnetic field makes no contribution to the torque.

We find after a straightforward calculation that the self-torque's only nonvanishing component is

$$
\mathcal{T}_{z}^{\mathrm{em}}=-\frac{\mu_{0} q}{6 \pi R}\left[(\dot{m}+\tau \ddot{m})+\frac{1}{10} \tau^{2}\left(m^{(3)}+\tau m^{(4)}\right)+\frac{1}{280} \tau^{4}\left(m^{(5)}+\tau m^{(6)}\right)+\frac{1}{15120} \tau^{6}\left(m^{(7)}+\tau m^{(8)}\right)+\cdots\right]_{t-R / c} .
$$

An alternative expression, with $m$ and its derivatives further expanded about the time $t$, is

$$
\mathcal{T}_{z}^{\mathrm{em}}=-\frac{\mu_{0} q}{6 \pi R}\left[\dot{m}-\frac{2}{5} \tau^{2} m^{(3)}+\frac{1}{3} \tau^{3} m^{(4)}-\frac{6}{35} \tau^{4} m^{(5)}+\frac{1}{15} \tau^{5} m^{(6)}-\frac{4}{189} \tau^{6} m^{(7)}+\frac{1}{175} \tau^{7} m^{(8)}+\cdots\right]_{t} .
$$

The expression of Eq. (4.1) is useful for the purpose of establishing angular momentum balance, as we do in Sec. VI. Equation (4.2) is a better starting point for the calculation of the angular motion of the spinning shell, which we present in Sec. VII It is also more convenient to single out the contributions associated with radiation reaction, as we do in the next paragraphs.

One would normally expect the self-force or self-torque acting on a distribution of charge to be associated with the electromagnetic radiation emitted by the distribution, and one would attach the words "radiation reaction" to the phenomenon. Our result for the self-torque reveals, however, that this expectation is not entirely fulfilled: the self-torque is not the sole result of radiation reaction. In fact, while radiation reaction does contribute, it is not the dominant contribution. 
The radiation-reaction terms in Eq. (4.2) can be identified as those that come with an odd power of $c$ (recall that $\tau=R / c)$; the terms that come with even powers of $c$ have nothing to do with radiation reaction. To understand this, recall that the fields of Sec. III were calculated on the basis of the retarded Green's function. These fields describe an outgoing electromagnetic wave that carries angular momentum outward, with the radiated loss of angular momentum balanced by a contribution to the self-torque. Suppose, however, that we had adopted the advanced solution instead of the retarded solution. In this case we would have had an incoming wave that carries angular momentum inward, and the radiated gain of angular momentum would have been balanced by an equal and opposite contribution to the self-torque. The advanced solution can be obtained from the retarded solution by the formal switch $c \rightarrow-c$, and the contributions to the self-torque that are sensitive to the choice of solution are therefore those that are odd in $c$; these are the radiation-reaction terms. The terms that are even in $c$ are the same regardless of the choice of solution, and these contributions to the self-torque - though they still have to do with the electromagnetic field created by the spinning sphere - have nothing to do with radiation reaction.

Another way of stating all this is to say that the complete self-torque is a sum of time-antisymmetric terms (odd in $c$ ) that make up the radiation-reaction piece, and time-symmetric terms (even in $c$ ) that have nothing to do with radiation reaction. The notions of self-torque and radiation reaction must therefore be distinguished.

Inspection of Eq. (4.2) reveals that the leading terms involving $\dot{m}$ and $m^{(3)}$ come with even powers of $c$ and are therefore time-symmetric; these contributions to the self-torque have nothing to do with radiation reaction. The leading radiation-reaction term occurs at order $\tau^{3}$ and is proportional to $m^{(4)}$.

\section{RADIATED ANGULAR MOMENTUM}

The radiated flux of angular momentum is calculated on the basis of Eq. (2.7). We choose $S$ to be a sphere of radius $r_{0}$, and we take $r_{0}$ to be very large compared with $\lambda_{c}$, a characteristic wavelength of the electromagnetic radiation. With $t_{c}$ denoting the characteristic time scale of variation of the magnetic moment (this quantity was introduced in Sec. III), we have that $\lambda_{c}=c t_{c}$, and we therefore assume that $r_{0} \gg c t_{c} \gg R$.

After inserting Eq. (2.2) and writing $d S_{a}=\hat{r}_{a} r_{0}^{2} d \Omega$ for the surface element (where $d \Omega:=\sin \theta d \theta d \phi$ ), we find that the angular-momentum flux can be expressed as

$$
\mathcal{N}_{a}^{\mathrm{em}}=-\epsilon_{0} \int r_{0}^{3}(\hat{\boldsymbol{r}} \cdot \boldsymbol{E})(\hat{\boldsymbol{r}} \times \boldsymbol{E})_{a} d \Omega-\frac{1}{\mu_{0}} \int r_{0}^{3}(\hat{\boldsymbol{r}} \cdot \boldsymbol{B})(\hat{\boldsymbol{r}} \times \boldsymbol{B})_{a} d \Omega .
$$

We make the substitutions from Eqs. (3.14) and (3.16), neglect terms that are suppressed by powers of $\lambda_{c} / r_{0}$, perform the angular integration, and obtain that the only nonvanishing component of the radiated flux is

$$
\mathcal{N}_{z}^{\mathrm{em}}=\frac{\mu_{0} q}{6 \pi c}\left[\ddot{m}+\frac{1}{10} \tau^{2} m^{(4)}+\frac{1}{280} \tau^{4} m^{(6)}+\frac{1}{15120} \tau^{6} m^{(8)}+\cdots\right]_{t-r_{0} / c},
$$

where $m$ and its derivatives are evaluated at the time $t-r_{0} / c$; we recall that $\tau=R / c$. The expression is proportional to $q$ and linear in the magnetic moment; this indicates that the radiated angular momentum results from an interplay between the Coulomb and induction pieces of the electric field.

The radiated flux of Eq. (5.2) is associated with the retarded solution to Maxwell's equation. As was discussed in Sec. IV] the advanced solution can be obtained with the formal switch $c \rightarrow-c$, and the advanced version of the radiated flux would come with an overall minus sign, and would involve $m$ and its derivatives evaluated at the time $t+r_{0} / c$.

\section{FIELD ANGULAR MOMENTUM}

The self-torque of Eq. (4.1) and the radiated angular momentum of Eq. (5.2) come at the expense of the field's own angular momentum, as implied by Eq. (2.6). The angular momentum in the electromagnetic field can be calculated from Eq. (2.5), which we write in the form

$$
J_{a}^{\mathrm{em}}=\epsilon_{0} \int_{V}\left[(\boldsymbol{x} \cdot \boldsymbol{B}) E_{a}-(\boldsymbol{x} \cdot \boldsymbol{E}) B_{a}\right] d^{3} x
$$

or

$$
J_{a}^{\mathrm{em}}=\int_{0}^{r_{0}} \mathfrak{j}_{a} d r
$$


with

$$
\mathfrak{j}_{a}:=\epsilon_{0} r^{3} \int\left[(\hat{\boldsymbol{r}} \cdot \boldsymbol{B}) E_{a}-(\hat{\boldsymbol{r}} \cdot \boldsymbol{E}) B_{a}\right] d \Omega ;
$$

we recall that $r_{0}$ is the radius of the sphere $S$ bounding $V$. Substitution from Eqs. (3.13) and (3.15) and evaluation of the angular integral reveals that $\mathrm{j}_{a}=0$ when $r<R$. For $r>R$ we get from Eqs. (3.14) and (3.16) that the only nonvanishing component of $\mathfrak{j}_{a}$ is

$$
\begin{aligned}
\mathfrak{j}_{z}= & \frac{\mu_{0} q}{6 \pi}\left[\left(\frac{m}{r^{2}}+\frac{\dot{m}}{c r}+\frac{\ddot{m}}{c^{2}}\right)+\frac{1}{10} \tau^{2}\left(\frac{m^{(2)}}{r^{2}}+\frac{m^{(3)}}{c r}+\frac{m^{(4)}}{c^{2}}\right)+\frac{1}{280} \tau^{4}\left(\frac{m^{(4)}}{r^{2}}+\frac{m^{(5)}}{c r}+\frac{m^{(6)}}{c^{2}}\right)\right. \\
& \left.+\frac{1}{15120} \tau^{6}\left(\frac{m^{(6)}}{r^{2}}+\frac{m^{(7)}}{c r}+\frac{m^{(8)}}{c^{2}}\right)+\cdots\right]_{t-r / c} .
\end{aligned}
$$

This must be integrated with respect to $r$, and it is a fortunate circumstance that each term in $\mathfrak{j}_{z}$ is a derivative with respect to $r$. For example,

$$
\frac{m}{r^{2}}+\frac{\dot{m}}{c r}+\frac{\ddot{m}}{c^{2}}=-\frac{\partial}{\partial r}\left(\frac{m}{r}+\frac{\dot{m}}{c}\right)
$$

where the partial derivative holds $t$ fixed. All remaining terms can be written in a similar way. The integration is performed from $r=R$ to $r=r_{0}$, and after neglecting terms that are suppressed by powers of $\lambda_{c} / r_{0}$, we arrive at

$$
\begin{aligned}
J_{z}^{\mathrm{em}}= & \frac{\mu_{0} q}{6 \pi R}\left[(m+\tau \dot{m})+\frac{1}{10} \tau^{2}\left(m^{(2)}+\tau m^{(3)}\right)+\frac{1}{280} \tau^{4}\left(m^{(4)}+\tau m^{(5)}\right)+\frac{1}{15120} \tau^{6}\left(m^{(6)}+\tau m^{(7)}\right)+\cdots\right]_{t-R / c} \\
& -\frac{\mu_{0} q}{6 \pi c}\left[\dot{m}+\frac{1}{10} \tau^{2} m^{(3)}+\frac{1}{280} \tau^{4} m^{(5)}+\frac{1}{15120} \tau^{6} m^{(7)}+\cdots\right]_{t-r_{0} / c} .
\end{aligned}
$$

As indicated, the first set of terms is evaluated at the time $t-R / c$, while the second set is evaluated at $t-r_{0} / c$. The first set can be thought of as the contribution to the angular momentum coming from the near-zone fields in the vicinity of the spinning shell. The second set can be thought of as the contribution from the wave-zone fields.

Differentiation of Eq. (6.6) with respect to $t$ and comparison with Eqs. (4.1) and (5.2) reveals that the field angular momentum satisfies $d J_{a}^{\mathrm{em}} / d t=-\mathcal{N}_{a}^{\mathrm{em}}-\mathcal{T}_{a}^{\mathrm{em}}$. This is Eq. (2.6), the statement of angular momentum balance that was first established in Sec. [II It is deeply satisfying that the statement can be verified explicitly.

\section{SHELL MOTION}

The self-torque of Eq. (4.2) affects the angular motion of the shell. The motion is governed by Eq. (2.14), $d J_{a}^{\text {mech }} / d t=\mathcal{T}_{a}^{\text {ext }}+\mathcal{T}_{a}^{\text {em }}$, where $J_{a}^{\text {mech }}$ is the shell's mechanical angular momentum, $\mathcal{T}_{a}^{\text {ext }}$ the torque supplied by an external agent, and $\mathcal{T}_{a}^{\mathrm{em}}$ the self-torque. In this section we explore the consequences of this equation.

\section{A. Equation of angular motion}

We take the shell to have a uniform mass density, and its mechanical angular momentum is related to the magnetic moment by

$$
\boldsymbol{J}^{\text {mech }}=\frac{2 M}{q} \boldsymbol{m},
$$

where $M$ is the shell's total mass. The shell's equation of motion is therefore

$$
\frac{2 M}{q} \dot{m}=\mathcal{T}^{\mathrm{ext}}-\frac{\mu_{0} q}{6 \pi R}\left(\dot{m}-\frac{2}{5} \tau^{2} m^{(3)}+\frac{1}{3} \tau^{3} m^{(4)}-\frac{6}{35} \tau^{4} m^{(5)}+\frac{1}{15} \tau^{5} m^{(6)}-\frac{4}{189} \tau^{6} m^{(7)}+\frac{1}{175} \tau^{7} m^{(8)}+\cdots\right),
$$

where $\mathcal{T}^{\text {ext }}$ is the $z$-component of the external torque (we assume that the remaining components vanish). We transfer the $\dot{m}$ term on the right-hand side of the equation to the other side, and write the combination as $\left(2 M_{\text {eff }} / q\right) \dot{m}$, where $M_{\mathrm{eff}}:=M+\delta M$ is an effective mass, with an electrostatic correction given by

$$
\delta M c^{2}=\frac{q^{2}}{12 \pi \epsilon_{0} R} .
$$


This contribution to the effective mass can be thought of as additional inertia provided by the electromagnetic field. Curiously, $\delta M c^{2}$ is a factor $2 / 3$ smaller than the electrostatic energy of a hollow sphere, given by $U=q^{2} /\left(8 \pi \epsilon_{0} R\right)$.

Introducing the notation

$$
\mathrm{T}:=\frac{q}{2 M_{\mathrm{eff}}} \mathcal{T}^{\mathrm{ext}}, \quad \varepsilon:=\frac{1}{M_{\mathrm{eff}} c^{2}} \frac{q^{2}}{30 \pi \epsilon_{0} R}=\frac{2}{5} \frac{\delta M}{M_{\mathrm{eff}}},
$$

we put the shell's equation of motion in the form

$$
\dot{m}=\mathrm{T}+\varepsilon\left(\tau^{2} m^{(3)}-\frac{5}{6} \tau^{3} m^{(4)}+\frac{3}{7} \tau^{4} m^{(5)}-\frac{1}{6} \tau^{5} m^{(6)}+\frac{10}{189} \tau^{6} m^{(7)}-\frac{1}{70} \tau^{7} m^{(8)}+\cdots\right),
$$

where, we recall, $\tau:=R / c$. We note that up to a factor of order unity, $\varepsilon$ is the ratio of the electrostatic contribution to the mass to the total effective mass. This ratio is small for a macroscopic body. To take the measure of this, we consider a sphere of mass $M=10 \mathrm{~g}$ and radius $R=1 \mathrm{~cm}$, on which we deposit a charge $q=0.1 \mathrm{C}$; for this object $\varepsilon \simeq 1 \times 10^{-6}$. The assumption placed on the variation of $m, \tau / t_{c} \ll 1$, ensures that $\tau^{2} m^{(3)} \ll \dot{m}, \tau^{3} m^{(4)} \ll \tau^{2} m^{(3)}$, and so on. The upshot is that the external term $T$ is strongly dominant in Eq. (7.5), and the self-torque terms provide small corrections. We recall that in Eq. (7.5), the terms with odd powers of $\tau$ are radiation-reaction terms; the terms with even powers of $\tau$ (including the leading-order term proportional to $m^{(3)}$ ) have nothing to do with radiation reaction.

Equation (7.5) contains high-order derivatives, and it would include terms of even higher order if the expansion in powers of $\tau / t_{c}$ were pursued beyond what is displayed there. Such equations are typically pathological and produce runaway solutions that strongly violate the underlying assumption that $\tau / t_{c} \ll 1$. A coping strategy presents itself on the grounds that $\varepsilon$ is small, and that each successive term contributes a smaller correction. The strategy is to perform a reduction of order, by substituting $\dot{m}=\mathrm{T}+O(\varepsilon)$ on the right-hand side of the equation. This yields

$$
\dot{m}=\mathrm{T}+\varepsilon\left(\tau^{2} \mathrm{~T}^{(2)}-\frac{5}{6} \tau^{3} \mathrm{~T}^{(3)}+\frac{3}{7} \tau^{4} \mathbf{T}^{(4)}-\frac{1}{6} \tau^{5} \mathrm{~T}^{(5)}+\frac{10}{189} \tau^{6} \mathbf{T}^{(6)}-\frac{1}{70} \tau^{7} \mathbf{T}^{(7)}+\cdots\right),
$$

where we neglect all terms of order $\varepsilon^{2}$ and higher 4

Equation (7.6) is free of pathologies, and its solution is immediate:

$$
m(t)=\int^{t} \mathrm{~T}\left(t^{\prime}\right) d t^{\prime}+\varepsilon\left(\tau^{2} \mathbf{T}^{(1)}-\frac{5}{6} \tau^{3} \mathbf{T}^{(2)}+\frac{3}{7} \tau^{4} \mathrm{~T}^{(3)}-\frac{1}{6} \tau^{5} \mathrm{~T}^{(4)}+\frac{10}{189} \tau^{6} \mathrm{~T}^{(5)}-\frac{1}{70} \tau^{7} \mathbf{T}^{(6)}+\cdots\right)+\text { constant },
$$

where the constant is determined by the initial condition. With $\mathrm{T}$ assumed to vary on a time scale $t_{c}$ that is long compared with $\tau=R / c$, Eq. (7.7) is guaranteed to describe a $m(t)$ that also varies on a long time scale.

\section{B. Examples}

For the purpose of illustration we examine a number of concrete examples.

To begin we consider the simplest case, that of a vanishing external torque. Setting $T=T_{1}:=0$ in Eq. (7.7) returns $m_{1}(t)=$ constant, and we see that as expected, the sphere rotates with a constant angular velocity when the external torque is zero. Equation (4.2) implies that the self-torque vanishes, Eq. (5.2) shows that there is no radiated flux of angular momentum, and Eq. (6.6) reveals that the field's angular momentum is $J^{\mathrm{em}}=\mu_{0} q m_{1} /(6 \pi R)$, a constant. In this rather trivial case, angular momentum is strictly conserved.

Next on our list of examples is that of a constant external torque, $T=T_{2}:=$ constant. In this case Eq. (7.7) returns $m_{2}(t)=m_{2}(0)+\mathrm{T}_{2} t$, and we find that the angular velocity increases linearly with time. Equation (4.2) implies that the self-torque is given by

$$
\mathcal{T}^{\mathrm{em}}=-\frac{\mu_{0} q}{6 \pi R} \mathrm{~T}_{2}=-\frac{\delta M}{M_{\mathrm{eff}}} \mathcal{T}^{\text {self }}=-\frac{5}{2} \varepsilon \mathcal{T}^{\text {self }},
$$

where we used Eqs. (7.3) and (7.4). This self-torque was already moved to the left-hand side of the equation of motion in Eq. (7.5), and shown to give rise to the shift $M \rightarrow M+\delta M=M_{\mathrm{eff}}$ in the sphere's mass. The previous equation

\footnotetext{
4 The reduction of order works even when $\varepsilon$ is taken to be of order unity. In this case the procedure relies entirely on the assumption that $\tau / t_{c} \ll 1$, and it produces a very similar form for the reduced equation of motion, with coefficients that acquire $\varepsilon$-dependent corrections.
} 
reveals another interpretation for the self-torque (valid in this specific case only): it produces a slight offset in the external torque. Equation (5.2) shows that there is no radiated flux of angular momentum.

Next we consider an external torque given by

$$
\mathrm{\top}=\mathrm{T}_{3}:=\mathfrak{m} k^{3} t^{2}
$$

where $\mathfrak{m}$ is a constant with unit of magnetic moment, and $k$ a constant with unit of inverse time. The solution to Eq. (7.7) is

$$
m_{3}(t)=m_{3}(0)+\frac{1}{3} \mathfrak{m}(k t)^{3}+2 \varepsilon \mathfrak{m} \zeta^{2}(k t)
$$

where $\zeta:=k \tau \ll 1$. In this case the magnetic moment is a cubic function of $t$. The leading term, growing as $t^{3}$, is contributed by the external torque, and the subleading term, proportional to $\varepsilon$ and growing as $t$, is contributed by the self-torque. It is interesting to note that the self-torque term is proportional to $\zeta^{2} \propto \tau^{2} \propto c^{-2}$ and therefore even in $c$. Recalling the discussion of Sec. IV] we conclude that the self-torque correction has nothing to do with radiation reaction. This is in spite of the fact that a magnetic moment that grows approximately as $t^{3}$ does radiate angular momentum, as shown by Eq. (5.2). The apparent paradox is resolved by noting that the radiated losses come at the expense of the field's angular momentum, and need not be balanced by a radiation-reaction contribution to the self-torque.

As a slight variation of the preceding example, we take

$$
\mathrm{T}=\mathrm{T}_{4}:=\mathfrak{m} k^{4} t^{3}
$$

where $\mathfrak{m}$ and $k$ are again constants. The corresponding solution to Eq. (7.7) is

$$
m_{4}(t)=m_{4}(0)+\frac{1}{4} \mathfrak{m}(k t)^{4}+\varepsilon \mathfrak{m}\left[3 \zeta^{2}(k t)^{2}-5 \zeta^{3}(k t)\right]
$$

where $\zeta:=k \tau \ll 1$. The leading term that grows as $t^{4}$ is contributed by the external torque, and the remaining terms are contributed by the self-torque. In this case we see that the self-torque correction contains a term proportional to $\zeta^{2}$, which is again even in $c$; this term has nothing to do with radiation reaction. But there is also a term proportional to $\zeta^{3}$, which is odd in $c$; this is a radiation-reaction contribution.

Next we consider an oscillating external torque given by

$$
\mathrm{T}=\mathrm{T}_{5}:=\mathfrak{m} \omega \cos \omega t
$$

where $\mathfrak{m}$ is a constant with unit of magnetic moment, and $\omega$ is a constant angular frequency. The corresponding solution to the equation of motion is

$$
\begin{aligned}
m_{5}(t)= & m_{5}(0)+\mathfrak{m}\left[1-\varepsilon\left(\zeta^{2}-\frac{3}{7} \zeta^{4}+\frac{10}{189} \zeta^{6}+\cdots\right)\right] \sin \omega t \\
& +\varepsilon \mathfrak{m}\left(\frac{5}{6} \zeta^{3}-\frac{1}{6} \zeta^{5}+\frac{1}{70} \zeta^{7}+\cdots\right)(\cos \omega t-1),
\end{aligned}
$$

where $\zeta:=\omega \tau \ll 1$. In the first set of terms, proportional to sin $\omega t$, the self-torque produces an amplitude correction that is even in $\zeta$; this correction is not to be associated with radiation reaction. The second set of terms is also contributed by the self-torque, and it represents a correction to the phase of the magnetic moment; these terms are odd in $\zeta$, and they are directly associated with radiation reaction.

For a final example we consider an external torque that gradually switches on and then gradually switches off. We describe it in terms of a gaussian function,

$$
\mathrm{\top}=\mathrm{T}_{6}:=\mathfrak{m} k e^{-k^{2} t^{2}}
$$

so that the external torque is active during a time interval proportional to $k^{-1}$ around $t=0$. The corresponding solution is

$$
\begin{aligned}
m_{6}(t)= & m_{6}(-\infty)+\frac{1}{2} \sqrt{\pi} \mathfrak{m}[\operatorname{erf}(k t)+1]-\varepsilon \mathfrak{m}\left\{2 \zeta^{2}(k t)+\frac{5}{3} \zeta^{3}\left[2(k t)^{2}-1\right]+\frac{12}{7} \zeta^{4}\left[2(k t)^{3}-3(k t)\right]\right. \\
& +\frac{2}{3} \zeta^{5}\left[4(k t)^{4}-12(k t)^{2}+3\right]+\frac{80}{189} \zeta^{6}\left[4(k t)^{5}-20(k t)^{3}+15(k t)\right]
\end{aligned}
$$




$$
\left.+\frac{4}{35} \zeta^{7}\left[8(k t)^{6}-60(k t)^{4}+90(k t)^{2}-15\right]+\cdots\right\} e^{-k^{2} t^{2}},
$$

where $\operatorname{erf}(u):=2 \pi^{-1 / 2} \int_{0}^{u} e^{-v^{2}} d v$ is the error function. The leading term in Eq. (7.16) describes a gradual increase of $m_{6}$ from its initial value $m_{6}(-\infty)$ to its final value $m_{6}(+\infty)=m_{6}(-\infty)+\sqrt{\pi}$ m. The corrections contributed by the self-torque are slight modulations superposed to the leading behavior described by the error function.

\section{ACKNOWLEDGMENTS}

Conversations with Bernie Nickel were greatly appreciated. This work was supported by the Natural Sciences and Engineering Research Council of Canada and by Perimeter Institute for Theoretical Physics. Research at Perimeter Institute is supported by the Government of Canada through the Department of Innovation, Science and Economic Development Canada and by the Province of Ontario through the Ministry of Research, Innovation and Science.

\section{Appendix A: Spherical-harmonic decomposition of the retarded Green's function}

The retarded Green's function for the wave equation is given by

$$
G\left(t, \boldsymbol{x} ; t^{\prime}, \boldsymbol{x}^{\prime}\right)=\frac{\delta\left(t-t^{\prime}-\left|\boldsymbol{x}-\boldsymbol{x}^{\prime}\right| / c\right)}{\left|\boldsymbol{x}-\boldsymbol{x}^{\prime}\right|} .
$$

Alternatively, it can be expressed as

$$
G\left(t, \boldsymbol{x} ; t^{\prime}, \boldsymbol{x}^{\prime}\right)=2 c \Theta\left(t-t^{\prime}\right) \delta\left[c^{2}\left(t-t^{\prime}\right)^{2}-\left|\boldsymbol{x}-\boldsymbol{x}^{\prime}\right|^{2}\right]
$$

with $\Theta(u)$ denoting the step function.

We wish to express the Green's function as a spherical-harmonic decomposition. We write

$$
G\left(t, \boldsymbol{x} ; t^{\prime}, \boldsymbol{x}^{\prime}\right)=\sum_{\ell \mathrm{m}} g_{\ell}\left(t, r ; t^{\prime}, r^{\prime}\right) Y_{\ell \mathrm{m}}^{*}\left(\theta^{\prime}, \phi^{\prime}\right) Y_{\ell \mathrm{m}}(\theta, \phi)
$$

where $g_{\ell}\left(t, r ; t^{\prime}, r^{\prime}\right)$ is a reduced Green's function for each multipole order $\ell$; the label $m$ does not appear because the reduced wave equation satisfied by $g_{\ell}$ is independent of $\mathrm{m}$. The strategy to obtain $g_{\ell}$ is to subject Eq. (A2) to a projection to each one of its multipole components.

We introduce the notation $\Delta:=c\left(t-t^{\prime}\right), s:=\left|\boldsymbol{x}-\boldsymbol{x}^{\prime}\right|$, and express Eq. (A2) in the condensed form $G=$ $2 c \Theta(\Delta) \delta\left(\Delta^{2}-s^{2}\right)$. We substitute this on the left-hand side of Eq. (A33), multiply each side by $Y_{\ell^{\prime} m^{\prime}}\left(\theta^{\prime}, \phi^{\prime}\right)$, and integrate over $d \Omega^{\prime}=\sin \theta^{\prime} d \theta^{\prime} d \phi^{\prime}$. The result is

$$
2 c \Theta(\Delta) \int \delta\left(\Delta^{2}-s^{2}\right) Y_{\ell \mathrm{m}}\left(\theta^{\prime}, \phi^{\prime}\right) d \Omega^{\prime}=g_{\ell} Y_{\ell \mathrm{m}}(\theta, \phi) .
$$

We next set $\mathrm{m}=0$ and use the fact that $Y_{\ell 0}(\theta, \phi) \propto P_{\ell}(\cos \theta)$. The previous equation reduces to

$$
2 c \Theta(\Delta) \int \delta\left(\Delta^{2}-s^{2}\right) P_{\ell}\left(\cos \theta^{\prime}\right) d \cos \theta^{\prime} d \phi^{\prime}=g_{\ell} P_{\ell}(\cos \theta)
$$

Finally, we set $\cos \theta=1$ and use the fact that $P_{\ell}(1)=1$. This gives

$$
g_{\ell}=\left.2 c \Theta(\Delta) \int \delta\left(\Delta^{2}-s^{2}\right)\right|_{\cos \theta=1} P_{\ell}\left(\cos \theta^{\prime}\right) d \cos \theta^{\prime} d \phi^{\prime} .
$$

Because $s^{2}$ evaluated at $\cos \theta=1$ is $r^{2}-2 r r^{\prime} \cos \theta^{\prime}+r^{\prime 2}$, we have that

$$
g_{\ell}=4 \pi c \Theta(\Delta) \int \delta\left(\Delta^{2}-r^{2}+2 r r^{\prime} \cos \theta^{\prime}-r^{2}\right) P_{\ell}\left(\cos \theta^{\prime}\right) d \cos \theta^{\prime},
$$

or

$$
g_{\ell}=\frac{2 \pi c \Theta(\Delta)}{r r^{\prime}} \int \delta\left(\cos \theta^{\prime}-\xi\right) P_{\ell}\left(\cos \theta^{\prime}\right) d \cos \theta^{\prime}
$$


where $\xi:=\left(r^{2}+r^{\prime 2}-\Delta^{2}\right) /\left(2 r r^{\prime}\right)$.

The integral is nonzero whenever $\xi$ lies in the interval between -1 and +1 ; when this condition is satisfied it evaluates to $g_{\ell}=2 \pi c \Theta(\Delta) P_{\ell}(\xi) /\left(r r^{\prime}\right)$. The condition $-1<\xi$ implies $-2 r r^{\prime}<r^{2}+r^{\prime 2}-\Delta^{2}$, so that $\Delta<r+r^{\prime}$. The condition $\xi<1$ implies $2 r r^{\prime}>r^{2}+r^{\prime 2}-\Delta^{2}$, so that $\Delta>\left|r-r^{\prime}\right|$. This last condition supersedes the requirement that $\Delta>0$, which comes from the step function appearing in $G$. Altogether, we find that the reduced Green's function is given by

$$
g_{\ell}\left(t, r ; t^{\prime}, r^{\prime}\right)=\frac{2 \pi c}{r r^{\prime}} \Theta\left(\Delta-\left|r-r^{\prime}\right|\right) \Theta\left(r+r^{\prime}-\Delta\right) P_{\ell}(\xi)
$$

The temporal support of the reduced Green's function is the interval $\left|r-r^{\prime}\right|<\Delta<r+r^{\prime}$.

[1] D. J. Griffiths, Introduction to electrodynamics. Fourth edition (Pearson, Boston, 2013).

[2] R. H. Romer, Angular momentum of static electromagnetic fields, Am. J. Phys. 34, 772-778 (1966).

[3] F. L. Boos, Jr, More on the Feynman's disk paradox, Am. J. Phys. 52, 756-757 (1984).

[4] R. P. Feynman, R. B. Leighton, and M. Sands, The Feynman Lectures on Physics, The New Millennium Edition (Basic Books, USA, 2011).

[5] G. G. Lombardi, Feynman's disk paradox, Am. J. Phys. 51, 213-214 (1983).

[6] J. D. Jackson, Classical Electrodynamics, Third Edition (Wiley, New York, 1999).

[7] T. H. Boyer, Mass renormalization and radiation damping for a charged particle in uniform circular motion, Am. J. Phys. 40, 1843-1846 (1972).

[8] F. Rohrlich, The dynamics of a charged sphere and the electron, Am. J. Phys. 65, 1051-1056 (1997).

[9] D. J. Griffiths, T. C. Proctor, and D. F. Schroeter, Abraham-Lorentz versus Landau-Lifshitz, Am. J. Phys. 78, 391-402 (2010).

[10] L. D. Landau and E. M. Lifshitz, The Classical Theory of Fields, Fourth Edition (Butterworth-Heinemann, Oxford, England, 2000).

[11] D. J. Griffiths and R. E. Owen, Mass renormalization in classical electrodynamics, Am. J. Phys. 51, 1120-1126 (1983).

[12] D. J. Griffiths and E. W. Szeto, Dumbbell model for the classical radiation reaction, Am. J. Phys. 46, 244-248 (1978).

[13] A. Ori and E. Rosenthal, Universal self force from an extended-object approach, Phys. Rev. D 68, 041701 (2003), arXiv:grqc/0205003.

[14] A. Ori and E. Rosenthal, Calculation of the self force using the extended-object approach, J. Math. Phys. 45, 2347-2364 (2004), arXiv:gr-qc/0309102.

[15] S. E. Gralla, A. I. Harte, and R. M. Wald, Rigorous derivation of electromagnetic self-force, Phys. Rev. D 80, 024031 (2009), arXiv:0905.2391.

[16] A. S. de Castro, Electromagnetic angular momentum for a rotating charged shell, Am. J. Phys. 59, 180-181 (1991).

[17] D. R. Stump and G. L. Pollack, Magnetic dipole oscillations and radiation damping, Am. J. Phys. 65, 81-87 (1997).

[18] A. Ashtekar and B. Bonga, On the ambiguity in the notion of transverse traceless modes of gravitational waves, Gen. Rel. Grav. 49, 122 (2017), arXiv:1707.09914.

[19] R. J. Cook, Radiation reaction revisited, Am. J. Phys. 52, 894-895 (1984). 